\title{
Prognostic value of gross tumor volume delineated by FDG-PET-CT based radiotherapy treatment planning in patients with locally advanced pancreatic cancer treated with chemoradiotherapy
}

\author{
Cem Parlak ${ }^{*}$, Erkan Topkan¹, Cem Onal', Mehmet Reyhan² and Ugur Selek ${ }^{3}$
}

\begin{abstract}
Background: We aimed to assess whether gross tumor volume (GTV) determined by fusion of contrast-enhanced computerized tomography (CT) and 18F-fluoro-deoxy-D-glucose positron emission tomography-CT (FDG-PET-CT) based radiotherapy planning could predict outcomes, namely overall survival (OS), local-regional progression-free survival (LRPFS), and progression-free survival (PFS) in cases with locally advanced pancreas cancer (LAPC) treated with definitive concurrent chemoradiotherapy.

Methods: A total of 30 patients with histological proof of LAPC underwent $50.4 \mathrm{~Gy}$ (1.8 Gy/28 fractions) of radiotherapy concurrent with continuously infused 5-FU followed by 4 to 6 courses of maintenance gemcitabine. Target volume delineations were performed on FDG-PET-CT-based RTP. Patients were stratified into 2 groups: GTV lesser $\left(G T V_{L}\right)$ versus greater $\left(G_{T} V_{G}\right)$ than cut off value determined by receiver operating characteristic (ROC) analysis, and compared in terms of OS, LRPFS and PFS.

Results: Median GTV delineated according to the FDG-PET-CT data was $100.0 \mathrm{~cm}^{3}$. Cut off GTV value determined from ROC curves was $91.1 \mathrm{~cm}^{3}$. At a median follow up of 11.2 months, median OS, LRPFS and PFS for the entire population were 10.3, 7.8 and 5.7 months, respectively. Median OS, LRPFS and PFS for GTV $\mathrm{L}$ and $\mathrm{GTV}_{\mathrm{G}}$ cohorts were 16.3 vs. 9.5 ( $p=0.005), 11.0$ vs. 6.0 ( $p=0.013$ ), and 9.0 vs. 4.8 months $(p=0.008)$, respectively.

Conclusions: The superior OS, LRPFS and PFS observed in GTV $V_{L}$ patients over GTV $V_{G}$ ones suggests a potential for FDG-PET-CT-defined GTV size in predicting outcomes of LAPC patients treated with definitive C-CRT, which needs to be validated by further studies with larger cohorts.
\end{abstract}

Keywords: Locally advanced pancreatic carcinoma, Chemoradiotherapy, FDG-PET-CT based RTP, Gross tumor volume

\section{Background}

Concurrent chemoradiotherapy (C-CRT) has been a well-established treatment option for patients with locally-advanced pancreas cancer (LAPC), which constitutes approximately one third of pancreatic carcinomas (PC) $[1,2]$. In spite of significant improvements in

\footnotetext{
* Correspondence: cemparlak@gmail.com

'Baskent University Adana Medical Faculty, Department of Radiation Oncology, Kisla Saglik Yerleskesi, Adana, Turkey

Full list of author information is available at the end of the article

diagnostic imaging, chemotherapy, and radiotherapy (RT), outcome in LAPC is still dismal with median overall survival (OS) rarely exceeding 1 year even following aggressive C-CRT $[3,4]$.

Several surgical series have established factors including tumor size, status of resection margins, invasion of vascular and/or adjacent structures, degree of differentiation, performance status, carbohydrate antigen 19-9 and C-reactive protein levels, to affect the outcome in resectable PC [2]. However, there are only few series 
indicating prognostic factors in the LAPC [4-7], while none - to the best of our knowledge- has specifically evaluated local/regional tumor burden in terms of gross tumor volume (GTV) in the era of definitive C-CRT, which was shown to be highly predictive for local control (LC), OS and progression-free survival (PFS) in various primaries including, lung, oral cavity and nasopharynx [8-11].

In studies by Lemke et al. [12] and Delbeke et al. [13] functional imaging with 18F-fluoro-deoxyglucose positron emission tomography (FDG-PET) has been demonstrated to increase accuracy of PC staging compared to conventional methods with its higher sensitivity and specificity. Based on these promising results, and considering the fact that accurate definition of primary tumor and its local/regional extensions is the first step of any effort to improve LC rates with $\mathrm{RT}$, in our earlier comparative dosimetric study, we demonstrated an average of $29.7 \%$ enlargement in GTV being necessary in 5 out of 14 (35.7\%) patients due to detection of additional computerized tomography-(CT) occult lymph node metastases and/or primary tumor extensions outlined by co-registered FDG-PET-CT [14]. From thereon, we changed our routine practice for target volume delineation that was based solely on contrast-enhanced CT findings, and began to use fusion of contrast-enhanced CT and FDGPET-CT for this purpose in patients with LAPC.

In this study, which involved the first 30 patients of our on-going phase II study targeting to enrol a total of 120 patients, we investigated whether size of GTV determined by contrast-enhanced CT/FDG-PET-CT fusion could predict treatment outcomes, namely OS, local-regional progression-free survival (LRPFS), and PFS in LAPC patients treated with C-CRT.

\section{Methods}

\section{Patients population}

Thirty consecutive patients, referred with histologically proven diagnosis of surgically unresectable LAPC from March 2008 to December 2010 were enrolled in this study. Our institutional definition for technically unresectable PC is to be stage III (T4N0-1 M0) disease, which is the involvement of celiac axis and/or superior mesenteric artery. Disease extent was determined in all patients with radiological studies and laparotomy or laparoscopy. Standard radiological studies included contrast-enhanced abdominal CT, magnetic resonance imaging (MRI) and/ or MR-cholangiopancreaticography (MRCP). All patients were re-staged via fusion of previous CT images (obtained $\leq 1$ week before PET/CT scans) with FDGPET-CT obtained for RTP. All patients underwent laparoscopic $(n=12)$ or laparotomic $(n=18)$ examination and biopsies for histologic diagnosis of primary tumor and enlarged/metabolically active regional lymph nodes and isolated single organ metastasis respecting the current standard institutional staging procedure for pancreatic carcinoma. Patients, who had received chemotherapy or abdominal irradiation previously, were not included in the study. The eligibility criteria also included an age of 18 to 70 years, Eastern Cooperative Oncology Group (ECOG) performance status (PS) of 0 to 2, presence of measurable or evaluable lesion, no contraindication for FDG-PET-CT imaging, an adequate bone marrow reserve (hemoglobin value of $\geq 10 \mathrm{~g} / \mathrm{dL}$, leucocyte of $\geq 4.000 \mu \mathrm{L}$, and thrombocyte of $\geq 100.000 \mu \mathrm{L}$ ), hepatic (aspartate aminotransferase or alanine aminotransferase of $<5$ times the upper limit) and renal function (serum creatinine $<2 \mathrm{mg} / \mathrm{dL}$ ). All patients signed informed consent, and the study design was approved by the Institutional Ethical Committee, in accordance with the Helsinki Declaration and Rules of Good Clinical Practice.

\section{FDG-PET-CT image registration and radiation treatment planning}

FDG-PET-CT scans were performed according to the institutional protocol described elsewhere [14]. Areas of FDG uptake were categorized as malignant based on location, intensity, shape, size, and visual correlation with contrast-enhanced CT images to differentiate physiologic from pathologic uptake.

Image registration and RTP were performed via Eclipse 7.5 (Varian Medical Systems, Palo Alto, CA, USA) RTP system. The use of standard uptake values (SUV) alone for determining malignant involvement for delineation is subjective; therefore SUV measurements were not specifically used alone for delineation. For all 30 patients, two radiation oncologists consensually defined the target volumes, with the assistance of a nuclear medicine physician, and contoured the GTV, the planning target volume (PTV), and the organs at risk (OAR) on the contrast-enhanced CT/FDG-PET-CT fusion images. While the GTV (primary and nodal) was delineated on the FDG-PET/CT fusion, all OAR volumes were contoured from the CT due to inherent difficulty with edge detection in contouring PET volumes. For each patient, GTV included the primary tumor $\left(G_{T V}\right)$ and involved lymph nodes $\left(G_{T V}\right)$ apparent on contrast-enhanced CT (short axis $\geq 1.5 \mathrm{~cm}$ ) and/or PET images. Those nodes $<1.5 \mathrm{~cm}$ were involved in GTV only if they were judged to be malignant on PET scan. Based on the literature [15] depicting nearly $1.5 \mathrm{~cm}$ movement of pancreas with respiration and considering the unavailability of motion tracking system and image guidance at our department, PTV was defined by adding 2 $\mathrm{cm}$ to GTV at all directions except for intersecting OAR restrictions to allow for microscopic extension, organ motion and set-up errors. Elective nodal irradiation was not permitted in the study. 


\section{Treatment delivery}

A single target volume with no cone down volumes was intended to be treated. A four-field technique (posteroanterior, antero-posterior, and laterals) was mandated, and treatment volumes were defined by using customized multi-leaf collimators. All patients received the RT protocol utilizing $18 \mathrm{MV}$ photon energy linear-accelerators. A dose of 50.4 Gy $(1.8 \mathrm{~Gy} / \mathrm{fr}$ ) was prescribed to encompass the defined PTV with isodose lines between $95 \%$ and $107 \%$. To achieve this, we used dosimetric practice wedges to modify beams. Dose-volume histograms were generated for each patient to assess target volume coverage and organ at risk doses. The maximum dose limits for normal tissues were 45 Gy for spinal cord; 50 Gy for small bowel and stomach; 50 Gy for $\leq$ one-third, 35 Gy for two-thirds, and $30 \mathrm{~Gy}$ for three-thirds of the liver; and $20 \mathrm{~Gy}$ for at least two-thirds of one functioning kidney.

All patients received continuously infused 5-FU (225 $\mathrm{mg} / \mathrm{m}^{2} /$ day, 7 days/week) throughout the RT course (for 5.5 weeks), and additional 4 to 6 courses of maintenance gemcitabine $\left(1,000 \mathrm{mg} / \mathrm{m}^{2}\right.$ IV over $100 \mathrm{~min}$, days 1 and 8 , every 21 days) following C-CRT.

\section{Toxicity assessment}

Patients were assessed weekly or if necessary more frequently during C-CRT, every 3 months for the first 2 year, and every 6 months, thereafter. Early and late clinical toxicity was recorded and blood was collected for haematology and biochemistry assays at each assessment. Toxicity was assessed and scored with the aid of CTC 3.0 (Common Toxicity Criteria).

\section{Response evaluation and follow-up}

Response to treatment was assessed by re-staging FDGPET-CT scans, carried out 12 weeks after the completion of the treatment, according to EORTC-1999 guidelines [16]. Thereafter, patients were monitored by $8-12$ weekly studies (blood count/chemistry; serum CEA and CA 19-9). Additional abdominal ultrasound and/or CT, chest CT, cranial magnetic resonance imaging, and FDG-PET-CT were used as indicated.

\section{Statistical analyses}

The primary aim of this study was to evaluate predictive usefulness of GTV on clinical outcomes. For this purpose, we used receiver operating characteristic (ROC) analysis to determine whether GTV improved discrimination of outcomes. The ROC analysis represents the area under the curve (AUC) of sensitivity versus false-positive rate (1specificity), and is equivalent to the probability that a predictive model will assign a higher probability of an event to subjects who subsequently have an event. Then, patients were dichotomized into two groups, GTV greater
$\left(G_{T V}\right)$ versus lesser $\left(G_{T} V_{L}\right)$ than the cut off value, and compared in terms of LRPFS, PFS, and OS. LRPFS was defined as survival without local-regional failure, calculated as the time between the first day of treatment and the date of local-regional failure or death/last visit. PFS and OS were calculated as the time between the first day of treatment and any type of disease progression, and the date of death/last visit, respectively. Survival analysis was performed using the Kaplan-Meier method, and survival curves were compared with two-sided log-rank tests. $P \leq$ 0.05 was considered statistically significant.

\section{Results}

Pretreatment characteristics of all 30 patients enrolled to the study are as summarized in the Table 1 . All cases received the prescribed dose of RT plus the scheduled chemotherapy concomitantly.

Median GTV delineated by utilizing the contrastenhanced CT/FDG-PET-CT fusion data was $100.0 \mathrm{~cm}^{3}$ (range, $32.9 \mathrm{~cm}^{3}$ to $224.3 \mathrm{~cm}^{3}$ ). From the ROC analysis, the best cut-off point was set at $91.1 \mathrm{~cm}^{3}$ where sensitivity

\section{Table 1 Patient characteristics}

\begin{tabular}{|c|c|}
\hline Characteristic & Value \\
\hline \multicolumn{2}{|l|}{ Age (Years) } \\
\hline Median & 57 \\
\hline Range & $39-68$ \\
\hline \multicolumn{2}{|l|}{ Gender (\%) } \\
\hline Male & $21(70)$ \\
\hline Female & $9(30)$ \\
\hline \multicolumn{2}{|c|}{ Performance Status (\%) } \\
\hline ECOG 0-1 & $23(76.7)$ \\
\hline ECOG 2 & $7(23.3)$ \\
\hline \multicolumn{2}{|c|}{ Location of Tumor (\%) } \\
\hline Head & $23(76.7)$ \\
\hline Body & $7(23.3)$ \\
\hline \multicolumn{2}{|c|}{ Clinical Stage (\%) } \\
\hline T4NO & $13(43.3)$ \\
\hline T4N1 & $17(56.7)$ \\
\hline \multicolumn{2}{|l|}{ SUVmax } \\
\hline Median & 14.5 \\
\hline Range & $6.2-22.6$ \\
\hline \multicolumn{2}{|l|}{ GTV $\left(\mathrm{cm}^{3}\right)$} \\
\hline Median & 100 \\
\hline Range & $32.3-224.3$ \\
\hline \multicolumn{2}{|l|}{$\mathrm{GTV}_{\mathrm{P}}\left(\mathrm{cm}^{3}\right)$} \\
\hline Median & 93.4 \\
\hline Range & $32.3-205.1$ \\
\hline \multicolumn{2}{|l|}{$\mathrm{GTV}_{\mathrm{N}}\left(\mathrm{cm}^{3}\right)$} \\
\hline Median & 7.7 \\
\hline Range & $0-19.2$ \\
\hline
\end{tabular}

Abbreviations: ECOG Eastern cooperative oncology group; GTV gross tumor volume; $G T V_{N}$ nodal gross tumor volume; $G T V_{P}$ primary gross tumor volume; SUV standard uptake value 
and specificity were $79.6 \%$ and of 91.7 , respectively. The area under the ROC curve was 77.7. In 13 cases (43.3\%), GTV was lower than $91.1 \mathrm{~cm}^{3}$.

At a median follow up of 11.2 months (range, 4.6 to 25.8 months), 23 out of 30 evaluable cases (76.7\%) died. Six cases were alive with no disease progression, 4 of whom were from the $G_{T V}$ group while remaining one was still alive with hepatic metastases. Median follow up was 14.1 months (range, 6.9 to 25.8 months) for $\mathrm{GTV}_{\mathrm{L}}$ and 9.5 months (range, 4.6 to 25.3 months) for $\mathrm{GTV}_{\mathrm{G}}$. Median OS, LRPFS, and PFS for the entire population were 10.3 months (95\% CI: 9.3 to 11.3 months), 7.8 months (95\% CI: 5.8 to 9.8 months), and 5.7 months (95\% CI: 4.6 to 6.7 months), respectively (Figure 1).

Comparisons of survival data by Log-rank test revealed that patients in group $\mathrm{GTV}_{\mathrm{L}}$ had significantly longer OS, LRPFS, and PFS than those in $\mathrm{GTV}_{\mathrm{G}}$ (Figure 2) $(p=0.005$, 0.013 and 0.008 , respectively). Corresponding median OS, LRPFS, and PFS for the cohorts $\mathrm{GTV}_{\mathrm{L}}$ versus $\mathrm{GTV}_{\mathrm{G}}$ were 16.3 [ $95 \% \mathrm{CI}, 11.6$ to 21.0 ] versus 9.5 ( $95 \% \mathrm{CI}, 8.0$ to 11.0 ), 11.0 (95\% CI, 2.6 to 19.4 ) versus 6.0 months (95\% CI, 4.1 to 7.9 ), and 9.0 ( $95 \% \mathrm{CI}, 0.8$ to 17.2 ) versus 4.8 (95\% CI, 3.1 to 6.5 ), respectively. Results of univariate analyses for OS, LRPFS, and PFS were given in Table 2.

During follow-up $24(80 \%)$ patients relapsed. Initial failure site was distant in 21 (70\%) and local in $3(10 \%)$ patients. The latter 3 patients also subsequently developed distant metastases during their follow up. Details of initial recurrence pattern according to the GTV groups are summarized in Table 3. Eventually, 16 (53.3\%) patients developed infield recurrences with accompanying distant

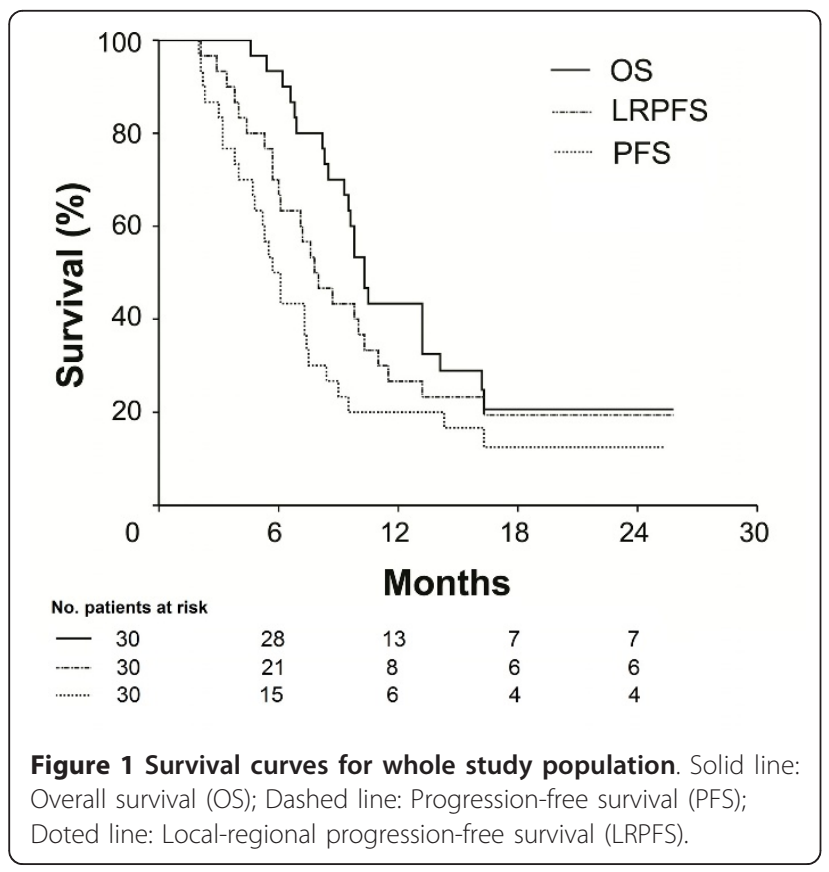

metastases. No isolated marginal or regional failure was reported as an initial or ultimate site of disease progression.

Rates of acute toxicities experienced in the study are listed in Table 4. In general, all 30 patients were able to tolerate the C-CRT with no Grade 4/5 acute toxicity. Unplanned treatment breaks was mandated in 4 patients (13.3\%) with an average of 3.8 days (range; 2 to 6 days), due to grade 3 toxicities; diarrhea in 2, leukopenia in 1, and vomiting in 1 patient. Because of Grade 3 diarrhea refractory to conventional loperamide and Grade 3 leukopenia, hospitalization was needed in 2 respective patients. However, all 4 patients were able to complete the planned C-CRT course after symptomatic and supportive treatment. At long-term, 3 patients (10.0\%) developed symptoms of grade 3 gastric outlet obstruction at median 5.1 months (range; 3.1 to 8.7 months) after completion of CCRT. Although late toxicity cannot be excluded as a cause, because of simultaneous evidence of disease progression in 2 patients at 4.4 and 7.1 months, progressive disease was probably associated with the symptoms in these two patients. Two additional (6.6\%) patients experienced grade 2 gastric ulcer at 10.6 and 14.7 months, respectively, which were successfully managed with appropriate medication. No patients developed liver or renal dysfunction attributed to this C-CRT protocol.

\section{Discussion}

We have investigated the predictive utility of GTV delineated by utilizing co-registered contrast-enhanced $\mathrm{CT} /$ FDG-PET-CT on outcomes in patients with LAPC treated with definitive C-CRT, and demonstrated that patients with smaller GTVs had significantly better OS, LRRFS and PFS compared to larger counterparts.

One of the reasons triggering our project is the impact of the size of the primary pancreatic tumors [17], which has been well-established in large surgical series of resectable PC demonstrating a better outcome with smaller sizes [18-20]. In their series, Birk et al. [18] and Sohn et al. [19] respectively demonstrated that patients with tumors < 2 and $<3 \mathrm{~cm}$ had significantly superior outcomes compared to those with $2-4$ and $\geq 3 \mathrm{~cm}$. In another study of 1697 patients from Johns Hopkins Hospital, de Jong et al. [20] also evaluated the impact of tumor size on survival following pancreaticoduodenectomy, and showed that 5year OS was inversely proportional to tumor size $(\leq 2 \mathrm{~cm}$ : $28.8 \%$ vs. $2-5 \mathrm{~cm}: 19.4 \%$ vs. $\geq 5 \mathrm{~cm}: 14.2 \%$, p < 0.001 ), and that the size correlated with the risk of other adverse factors, with larger tumors being more likely to be associated with nodal disease and poor differentiation $(p<0.05)$.

Although tumor size at largest dimension has been addressed in a limited number of studies [21-24], to the best of our knowledge, the impact of three dimensional local/regional tumor burden expressed as GTV on outcome 


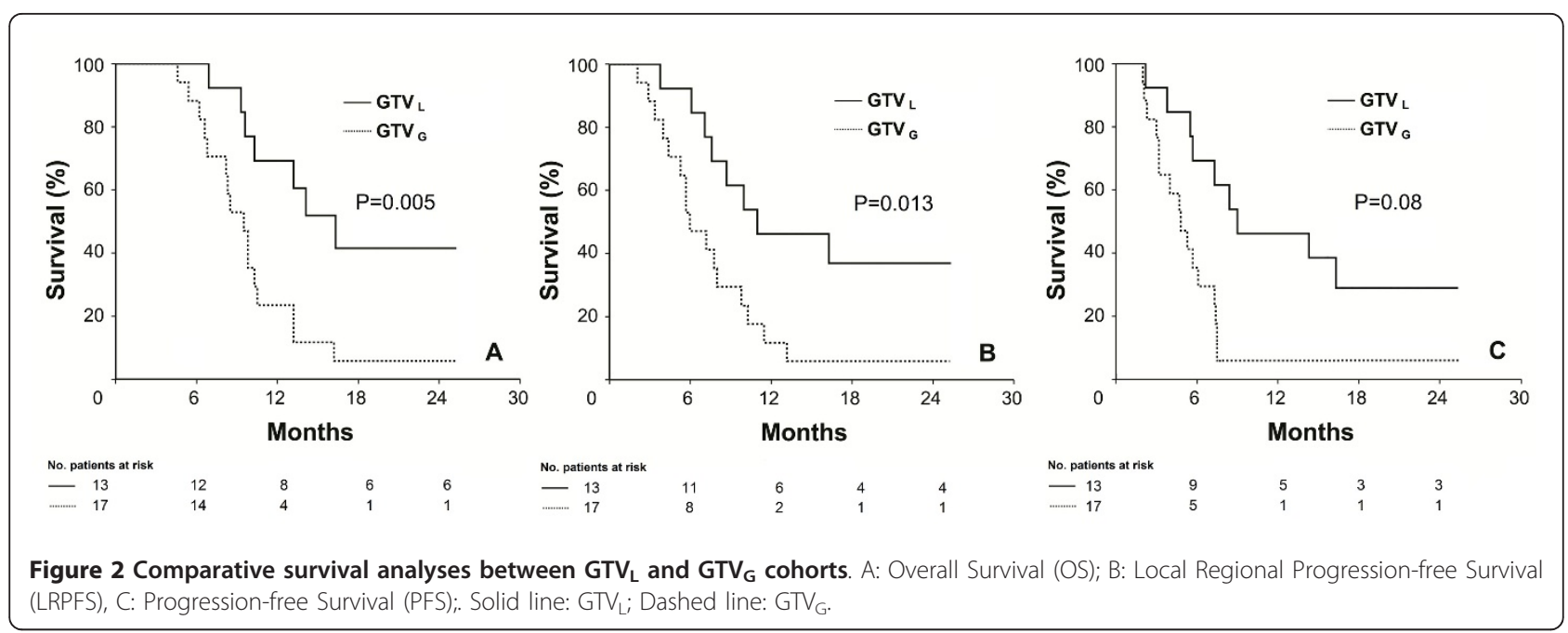

in the era of unresectable LAPC treated with definitive C-CRT has not been formally evaluated yet. In this respect, although two studies appeared to directly evaluate prognostic utility of the GTV size on outcome in LAPC patients $[25,26]$, our study differs from them in significant aspects. In the first study, Rwigema et al. [25] successfully treated a group of LAPC patients including primary, recurrent, resected but margin positive and metastatic disease with stereotactic body radiotherapy (SBRT). This was a true volumetric study similar to one presented here, and found $15 \mathrm{~mL}$ as a significant volumetric cut point for survival difference. In the second study reported recently, Bjerregaard et al. [26] evaluated the impact of GTV on outcomes of initially unresectable 176 patients with LAPC. In this study, the authors chose to group patients by $25 \mathrm{~cm}^{3}$ increments in GTV, and reported a survival advantage favoring the patients with smaller GTVs. However, although their results are in line with ours, there are

Table 2 Univariate analyses for survival

\begin{tabular}{|c|c|c|c|c|c|c|c|}
\hline \multirow[t]{2}{*}{ Characteristics } & \multirow[t]{2}{*}{$N$} & Median OS & \multirow[t]{2}{*}{$\mathbf{P}$} & Median LRPFS & \multirow[t]{2}{*}{$\mathbf{P}$} & Median PFS & \multirow[t]{2}{*}{$\mathbf{P}$} \\
\hline & & Months $(95 \% \mathrm{Cl})$ & & Months $(95 \% \mathrm{Cl})$ & & Months $(95 \% \mathrm{Cl})$ & \\
\hline \multicolumn{8}{|l|}{ GTV } \\
\hline $\mathrm{GTV}_{\mathrm{L}}$ & 13 & $16.3(11.6-21.0)$ & 0.005 & $11.0(2.6-19.4)$ & 0.013 & $9.0(0.8-17.2)$ & 0.008 \\
\hline $\mathrm{GTV}_{\mathrm{G}}$ & 17 & $9.5(8.0-11.0)$ & & $6.0(4.1-7.9)$ & & $4.8(3.1-6.5)$ & \\
\hline $\mathrm{GTV}_{\mathrm{N}}$ & & - & & & & & \\
\hline$<$ Median & 15 & $13.2(8.2-18.2)$ & 0.085 & $9.8(7.3-12.3)$ & 0.18 & $8.4(4.9-11.9)$ & 0.086 \\
\hline$\geq$ Median & 15 & $9.5(7.511 .5)$ & & $6.0(3.3-8.7)$ & & $4.8(3.2-6.4)$ & \\
\hline \multicolumn{8}{|c|}{ Nodal status } \\
\hline No & 13 & $13.2(7.8-18.6)$ & 0.25 & $9.8(7.5-12.1)$ & 0.36 & $8.4(5.2-11.6)$ & 0.25 \\
\hline N1 & 17 & $9.8(7.8-11.8)$ & & $6.1(4.1-8.1)$ & & $4.8(3.1-6.5)$ & \\
\hline \multicolumn{8}{|l|}{ Age } \\
\hline$<$ Median & 14 & $10.5(7.2-13.8)$ & 0,99 & $7.6(6.1-9.1)$ & 0.97 & $5.7(4.6-6.8)$ & 0.92 \\
\hline$\geq$ Median & 16 & $9.8(9.0-10.6)$ & & $7.8(2.5-13.1)$ & & $5.7(0.8-10.6)$ & \\
\hline \multicolumn{8}{|l|}{ Gender } \\
\hline Male & 21 & $10.3(9.0-11.6)$ & 0.86 & $7.6(6.3-8.9)$ & 0.52 & $5.7(4.5-6.9)$ & 0.89 \\
\hline Female & 9 & $10.3(8.8-11.8)$ & & $10.3(3.0-17.6)$ & & $7.3(2.6-12.0)$ & \\
\hline \multicolumn{8}{|l|}{ ECOG } \\
\hline $0-1$ & 23 & $10.3(9.2-11.4)$ & 0.61 & $8.0(5.7-10.3)$ & 0.84 & $5.3(3.1-7.5)$ & 0.88 \\
\hline 2 & 7 & $10.3(7.7-12.9)$ & & $7.6(6.3-8.9)$ & & $7.3(3.2-11.4)$ & \\
\hline \multicolumn{8}{|c|}{ Location of Tumor } \\
\hline Head & 23 & $10.3(9.1-11.5)$ & 0.58 & $7.6(4.6-10.6)$ & 0.52 & $5.7(4.8-6.6)$ & 0.31 \\
\hline Body & 7 & $10.5(8.7-12.3)$ & & $8.7(6.4-11.0)$ & & $7.3(2.2-12.4)$ & \\
\hline
\end{tabular}

Abbreviations: ECOG Eastern cooperative oncology group; $G T V$ gross tumor volume; $G_{T V}$ gross tumor volume greater than 91.1 ( $\mathrm{cm}^{3}$ ); $G T V_{L}$ gross tumor volume lesser than $91.1\left(\mathrm{~cm}^{3}\right) ; G T V_{N}$ nodal gross tumor volume; LRPFS local regional progression-free survival; OS overall survival; PFS progression-free survival 
Table 3 Patterns of initial disease progression $(\mathbf{N}=\mathbf{3 0})$

\begin{tabular}{|c|c|c|c|}
\hline Site & $\begin{array}{l}\mathrm{GTV}_{\mathrm{G}}(\mathrm{N}: 17) \\
\mathrm{N}(\%)\end{array}$ & $\begin{array}{l}\text { GTV }(N: 13) \\
N(\%)\end{array}$ & $\begin{array}{l}\text { Overall (N:30) } \\
N(\%)\end{array}$ \\
\hline Local & $3(17.6)$ & $0(0)$ & $3(10)$ \\
\hline Regional & $0(0)$ & $0(0)$ & $0(0)$ \\
\hline Distant & $13(76.5)$ & $8(61.5)$ & $21(70)$ \\
\hline Liver & $3(17.6)$ & $3(23.1)$ & $6(20.0)$ \\
\hline Peritoneum & $2(11.8)$ & $1(7.6)$ & $3(10.0)$ \\
\hline Brain & $0(0)$ & $1(7.6)$ & $1(3.3)$ \\
\hline Multiorgan & $8(47.1)$ & $3(23.1)$ & $11(36.7)$ \\
\hline Total progression & $16(94.1)$ & $8(61.5)$ & $24(80.0)$ \\
\hline
\end{tabular}

Abbreviations: $G T V_{G}$ gross tumor volume greater than $91.1\left(\mathrm{~cm}^{3}\right) ; G T V_{L}$ gross tumor volume lesser than $91.1\left(\mathrm{~cm}^{3}\right)$

remarkable differences between these two studies and the one presented here: First, Rwigema and colleagues used SBRT, which have significantly different radiobiological effects on tumor tissue than fractionated 3D conformal RT utilized here, and although our population size is relatively smaller, it is still more homogenous since we did not include resected, recurrent or metastatic patients. And second, Bjerregaard and colleagues studied on a highly heterogeneous study population, which included of 72 T3N0, 71 T3N1, and only 33 T4NX patients. Therefore, it is difficult to generalize their results for $\mathrm{T} 4 \mathrm{NO} 0-1$ patients as they constituted less than $20 \%$ of the study population. Likewise, authors chose to group patients by somehow arbitrarily specified $25 \mathrm{~cm}^{3}$ increments in GTV rather than using possibly more relevant ROC defined cut off point(s), which creates further difficulties in interpretation of outcomes.

Herein, we preferred 3D tumor volume to define the tumor burden based on the studies suggesting that onedimensional tumor measurements may not be as representative of tumor size as true volume calculations, especially in irregularly shaped tumors. In patients with advanced head and neck cancer, Rudat et al. [27] reported precision and reliability of the CT based volume measurements by repeated measurements using irregularly shaped

Table 4 Frequency of Grade 3 acute toxicities

\begin{tabular}{llll}
\hline Toxicity & \multicolumn{3}{c}{ Grade } \\
\cline { 2 - 4 } & $\mathbf{0 - 1}$ & $\mathbf{2}$ & $\mathbf{3}$ \\
\hline Nausea & 24 & 4 & 2 \\
Vomiting & 26 & 3 & 1 \\
Diarrhea & 24 & 4 & 2 \\
Tumor pain & 28 & 2 & 0 \\
Anorexia & 20 & 10 & 0 \\
Fatigue & 26 & 3 & 1 \\
Gastritis & 28 & 2 & 0 \\
Leukopenia & 21 & 7 & 2 \\
Anemia & 23 & 6 & 1 \\
Thrombocytopenia & 24 & 5 & 1 \\
\hline
\end{tabular}

phantoms. Furthermore, Titola et al. [28] proposed that one-dimensional volume estimation of irregularly shaped tumor-like phantoms should be substituted by true computer-based volume calculations.

The GTV, investigated here, has also been found to be of highly prognostic significance in various tumor sites. Bradley et al. [8] reported GTV to be a prognostic factor on both univariate and multivariate analysis in 207 NSCLC patients treated with definitive 3D conformal RT with or without chemotherapy, while Etiz et al. [9] pointed out the importance of total tumor volume $\left(<\right.$ or $\left.\geq 80 \mathrm{~cm}^{3}\right)$, defined by combination of primary tumor and nodal volume, on OS in irradiated inoperable NSCLC patients. Predictive role of GTV, delineated by 3D conformal RTP, on outcomes has also been demonstrated in nasopharyngeal [10], hypopharyngeal $[29,30]$ and other head and neck sites $[11,31,32]$. Likewise, we have studied to pioneer the importance of well-defined GTV in the setting of LAPC, and our results revealed that median OS, LRRFS and PFS for the cohort with GTV lower than and greater than the ROC-defined $91.1 \mathrm{~cm}^{3}$ cut value were 14.1 versus 9.5 months, 10.0 versus 6.0 months, and 8.4 versus 4.8 months, respectively ( $p<0.05$ for each).

Based on the assumption that the majority of the benefit from RT would result from control of the primary tumor, rather than subclinical disease in lymph nodes, which could potentially be controlled by the systemic chemotherapy, as would more distant sites, we did not electively irradiate uninvolved regional nodes. Supporting this assumption we observed no isolated regional recurrences, which is consistent with findings of other studies which excluded elective nodal irradiation [33,34]. In a phase I radiation dose escalation study of 34 unresectable or incompletely resected PC patients treated with RT plus concurrent gemcitabine, McGinn et al. [33] reduced RT fields $(\mathrm{PTV}=\mathrm{GTV}+1 \mathrm{~cm}$ ) which was even smaller than our current definition, and observed only 3 (8.8\%) regional failures. The approach excluding the elective irradiation of regional nodes is further supported by the current radiosurgical practice in PC [34], which permits only milimetric margins around the GTV with loco-regional control and survival rates similar or even better to that of conventional larger field external beam RT studies. Taken together, we advocate the idea not to irradiate elective nodal irradiation at least to an attempt to decrease C-CRT-related toxicity and possibly to escalate the RT dose to more effective levels with highly conformal irradiation techniques until this issue is enlightened with randomized trials.

\section{Conclusions}

We have investigated the potential prognostic value of the GTV delineated by co-registered contrast-enhanced CT/FDG-PET-CT-based RTP on outcomes in patients with LAPC. The superior OS, LRPFS and PFS observed 
in $G T V_{L}$ patients over $G_{T V}$ ones suggests a potential for FDG-PET-CT-defined GTV size in predicting outcomes of LAPC patients treated with definitive C-CRT. Since it is neither easy nor appropriate to conclude firmly with findings from such a small study population, results presented here should better be interpreted with caution and warrants to be addressed in future trials with larger cohorts.

\section{Acknowledgements}

We would like to give our special thanks to Cagla Sariturk, PhD for her kind help during statistical analyses.

\section{Author details}

'Baskent University Adana Medical Faculty, Department of Radiation Oncology, Kisla Saglik Yerleskesi, Adana, Turkey. ${ }^{2}$ Baskent University Adana Medical Faculty, Department of Nuclear Medicine, Kisla Saglik Yerleskesi, Adana, Turkey. ${ }^{3}$ American Hospital- University of Texas M.D. Anderson Radiation Oncology Center, Istanbul, Turkey.

\section{Authors' contributions}

Study conception and design: CP, ET. Provision of study materials or patients: CP, ET, MR. Collection and assembly of data: CP, ET, CO. Data analysis and interpretation: $C P, E T, M R$, US. Manuscript writing: CP, ET, US. Final approval of manuscript: CP, ET, CO, MR, US. All authors read and approved the final manuscript.

\section{Competing interests}

We have no personal or financial competing interest and have not entered into any agreement that could interfere with our access to the research data, or upon our ability to analyze the data independently, to prepare manuscripts, or to publish them.

Received: 20 December 2011 Accepted: 19 March 2012 Published: 19 March 2012

\section{References}

1. Pliarchopoulou K, Pectasides D: Pancreatic cancer: current and future treatment strategies. Cancer Treat Rev 2009, 35(5):431-436.

2. Huguet F, Girard N, Guerche CS, Hennequin C, Mornex F, Azria D: Chemoradiotherapy in the management of locally advanced pancreatic carcinoma: a qualitative systematic review. J Clin Oncol 2009, 27(13):2269-2277.

3. Radiation therapy combined with Adriamycin or 5-fluorouracil for the treatment of locally unresectable pancreatic carcinoma. Gastrointestinal Tumor Study Group. Cancer 1985, 56(11):2563-2568.

4. Klaassen DJ, Maclntyre JM, Catton GE, Engstrom PF, Moertel CG: Treatment of locally unresectable cancer of the stomach and pancreas: a randomized comparison of 5 -fluorouracil alone with radiation plus concurrent and maintenance 5-fluorouracil-an Eastern Cooperative Oncology Group study. J Clin Oncol 1985, 3(3):373-378.

5. Ikeda M, Okada S, Tokuuye K, Ueno H, Okusaka T: Prognostic factors in patients with locally advanced pancreatic carcinoma receiving chemoradiotherapy. Cancer 2001, 91(3):490-495.

6. Krishnan S, Rana V, Janjan NA, Abbruzzese JL, Gould MS, Das P, Delclos ME, Palla S, Guha S, Varadhachary G, et al: Prognostic factors in patients with unresectable locally advanced pancreatic adenocarcinoma treated with chemoradiation. Cancer 2006, 107(11):2589-2596.

7. Papadoniou N, Kosmas C, Gennatas K, Polyzos A, Mouratidou D, Skopelitis E, Tzivras M, Sougioultzis S, Papastratis G, Karatzas G, et al: Prognostic factors in patients with locally advanced (unresectable) or metastatic pancreatic adenocarcinoma: a retrospective analysis. Anticancer Res 2008, 28(1B):543-549.

8. Bradley JD, leumwananonthachai N, Purdy JA, Wasserman TH, Lockett MA, Graham MV, Perez CA: Gross tumor volume, critical prognostic factor in patients treated with three-dimensional conformal radiation therapy for non-small-cell lung carcinoma. Int J Radiat Oncol Biol Phys 2002, 52(1):49-57.

9. Etiz D, Marks LB, Zhou SM, Bentel GC, Clough R, Hernando ML, Lind PA: Influence of tumor volume on survival in patients irradiated for nonsmall-cell lung cancer. Int J Radiat Oncol Biol Phys 2002, 53(4):835-846.

10. Lee CC, Huang TT, Lee MS, Hsiao SH, Lin HY, Su YC, Hsu FC, Hung SK: Clinical application of tumor volume in advanced nasopharyngeal carcinoma to predict outcome. Radiat Oncol 2010, 5:20.

11. Schinagl DA, Span PN, Oyen WJ, Kaanders JH: Can FDG PET predict radiation treatment outcome in head and neck cancer? Results of a prospective study. Eur J Nucl Med Mol Imaging 2011, 38(8):1449-1458.

12. Lemke AJ, Niehues SM, Hosten N, Amthauer H, Boehmig M, Stroszczynski C, Rohlfing T, Rosewicz S, Felix R: Retrospective digital image fusion of multidetector CT and 18 F-FDG PET: Clinical value in pancreatic lesions A retrospective study with 104 patients. J Nucl Med 2004, 45(8):1279-1286.

13. Delbeke D, Rose DM, Chapman WC, Pinson CW, Wright JK, Beauchamp RD, Shyr Y, Leach SD: Optimal interpretation of FDG PET in the diagnosis, staging and management of pancreatic carcinoma. J Nucl Med 1999, 40:1784-1791.

14. Topkan E, Yavuz AA, Aydin M, Onal C, Yapar F, Yavuz MN: Comparison of CT and PET-CT based planning of radiation therapy in locally advanced pancreatic carcinoma. J Exp Clin Cancer Res 2008, 27:41.

15. Gwynne S, Wills L, Joseph G, John G, Staffurth J, Hurt C, Mukherjee S: Respiratory movement of upper abdominal organs and its effect on radiotherapy planning in pancreatic cancer. Clin Oncol (R Coll Radiol) 2009, 21(9):713-719.

16. Young H, Baum R, Cremerius U, Herholz K, Hoekstra O, Lammertsma AA, Pruim J, Price P: Measurement of clinical and subclinical tumour response using [18 F]-fluorodeoxyglucose and positron emission tomography: review and 1999 EORTC recommendations. European Organization for Research and Treatment of Cancer (EORTC) PET Study Group. Eur J Cancer 1999, 35(13):1773-1782.

17. Vincent A, Herman J, Schulick R, Hruban RH, Goggins M: Pancreatic cancer. Lancet 2011, 378(9791):607-620.

18. Birk D, Fortnagel G, Formentini A, Beger HG: Small carcinoma of the pancreas. Factors of prognostic relevance. J Hepatobiliary Pancreat Surg 1998, 5(4):450-454.

19. Sohn TA, Yeo CJ, Cameron JL, Koniaris L, Kaushal S, Abrams RA, Sauter PK, Coleman J, Hruban RH, Lillemoe KD: Resected adenocarcinoma of the pancreas-616 patients: results, outcomes, and prognostic indicators. $J$ Gastrointest Surg 2000, 4(6):567-579.

20. de Jong MC, Li F, Cameron JL, Wolfgang CL, Edil BH, Herman JM, Choti MA, Eckhauser F, Hirose K, Schulick RD, et al: Re-evaluating the impact of tumor size on survival following pancreaticoduodenectomy for pancreatic adenocarcinoma. J Surg Oncol 2011, 103(7):656-662.

21. Prat F, Chapat O, Ducot B, Ponchon T, Fritsch J, Choury AD, Pelletier G, Buffet $C$ : Predictive factors for survival of patients with inoperable malignant distal biliary strictures: a practical management guideline. Gut 1998, 42(1):76-80.

22. Willett CG, Del Castillo CF, Shih HA, Goldberg S, Biggs P, Clark JW, Lauwers G, Ryan DP, Zhu AX, Warshaw AL: Long-term results of intraoperative electron beam irradiation (IOERT) for patients with unresectable pancreatic cancer. Ann Surg 2005, 241(2):295-299.

23. Katz A, Hanlon A, Lanciano R, Hoffman J, Coia L: Prognostic value of CA 19-9 levels in patients with carcinoma of the pancreas treated with radiotherapy. Int J Radiat Oncol Biol Phys 1998, 41(2):393-396.

24. Breslin $T M$, Hess KR, Harbison DB, Jean ME, Cleary KR, Dackiw AP, Wolff RA, Abbruzzese JL, Janjan NA, Crane CH, et al: Neoadjuvant chemoradiotherapy for adenocarcinoma of the pancreas: treatment variables and survival duration. Ann Surg Oncol 2001, 8(2):123-132.

25. Rwigema JC, Parikh SD, Heron DE, Howell M, Zeh H, Moser AJ, Bahary N, Quinn A, Burton SA: Stereotactic body radiotherapy in the treatment of advanced adenocarcinoma of the pancreas. Am J Clin Oncol 2011, 34(1):63-69.

26. Bjerregaard JK, Mortensen MB, Jensen HA, Nielsen M, Pfeiffer P: Prognostic factors for survival and resection in patients with initial nonresectable locally advanced pancreatic cancer treated with chemoradiotherapy. Int J Radiat Oncol Biol Phys 2011, doi:10.1016/j.ijrobp.2011.09.008.

27. Rudat V, Dietz A, Schramm O, Conradt C, Maier H, Flentje M, Wannenmacher M: Prognostic impact of total tumor volume and hemoglobin concentration on the outcome of patients with advanced 
head and neck cancer after concomitant boost radiochemotherapy. Radiother Oncol 1999, 53(2):119-125.

28. Tiitola M, Kivisaari L, Tervahartiala P, Palomaki M, Kivisaari RP, Mankinen P, Vehmas T: Estimation or quantification of tumour volume? CT study on irregular phantoms. Acta Radiol 2001, 42(1):101-105.

29. Tsou YA, Hua JH, Lin MH, Tsai MH: Analysis of prognostic factors of chemoradiation therapy for advanced hypopharyngeal cancer-does tumor volume correlate with central necrosis and tumor pathology? ORL J Otorhinolaryngol Relat Spec 2006, 68(4):206-212.

30. Chen SW, Yang SN, Liang JA, Lin FJ, Tsai MH: Prognostic impact of tumor volume in patients with stage III-IVA hypopharyngeal cancer without bulky lymph nodes treated with definitive concurrent chemoradiotherapy. Head Neck 2009, 31(6):709-716.

31. Seol YM, Kwon BR, Song MK, Choi YJ, Shin HJ, Chung JS, Cho GJ, Lee JC, Lee BJ, Wang SG, et al: Measurement of tumor volume by PET to evaluate prognosis in patients with head and neck cancer treated by chemo-radiation therapy. Acta Oncol 2010, 49(2):201-208.

32. Strongin A, Yovino S, Taylor R, Wolf J, Cullen K, Zimrin A, Strome S, Regine W, Suntharalingam M: Primary tumor volume is an important predictor of clinical outcomes among patients with locally advanced squamous cell cancer of the head and neck treated with definitive chemoradiotherapy. Int J Radiat Oncol Biol Phys 2011, doi:10.1016/j. ijrobp.2010.10.053.

33. MCGinn CJ, Zalupski MM, Shureiqi I, Robertson JM, Eckhauser FE, Smith DC, Brown D, Hejna G, Strawderman M, Normolle D, Lawrence TS: Phase I trial of radiation dose escalation with concurrent weekly full-dose gemcitabine in patients with advanced pancreatic cancer. J Clin Oncol. 2001, 19:4202-4208.

34. Chang DT, Schellenberg D, Shen J, Kim J, Goodman KA, Fisher GA, Ford JM, Desser T, Quon A, Koong AC: Stereotactic radiotherapy for unresectable adenocarcinoma of the pancreas. Cancer 2009, 115(3):665-672.

doi:10.1186/1748-717X-7-37

Cite this article as: Parlak et al:: Prognostic value of gross tumor volume delineated by FDG-PET-CT based radiotherapy treatment planning in patients with locally advanced pancreatic cancer treated with chemoradiotherapy. Radiation Oncology 2012 7:37.

\section{Submit your next manuscript to BioMed Central and take full advantage of:}

- Convenient online submission

- Thorough peer review

- No space constraints or color figure charges

- Immediate publication on acceptance

- Inclusion in PubMed, CAS, Scopus and Google Scholar

- Research which is freely available for redistribution

Submit your manuscript at www.biomedcentral.com/submit
Biomed Central 